ARTIGO DE REVISÃO

ISSN 1677-5090

(C) 2019 Revista de Ciências Médicas e Biológicas

DOI: http://dx.doi.org/10.9771/cmbio.v18i2.31849

\title{
Benefícios da utilização de bifidobactérias e lactobacilos no tratamento da colite ulcerativa: uma revisão
}

\author{
Benefits of the use of bifidobacteria and lactobacilos in the treatment of ulcerative \\ colitis: a review
}

\begin{abstract}
Eliane dos Santos da Conceição ${ }^{1}$, Maria Izabel Lamounier Vasconcelos ${ }^{2}$, Marcos da Costa Silva ${ }^{3 *}$
${ }^{1}$ Mestranda em Imunologia pela Universidade Federal da Bahia (UFBA).; ${ }^{2}$ Mestre em Ciências Farmacêuticas pela Universidade de São Paulo (USP).; ${ }^{3}$ Doutor em Imunologia pela UFBA, Professor da Universidade do Estado da
\end{abstract} Bahia (UNEB).

\begin{abstract}
Resumo
Introdução: a colite ulcerativa é uma doença crônica que ocasiona inflamação das mucosas do cólon, ceco e reto. Algumas evidências sugerem que o desenvolvimento e as recidivas da colite estão relacionadas à susceptibilidade genética, ao desequilíbrio da microbiota intestinal e à resposta anormal do sistema imunológico a antígenos entéricos. Diante disso, a modulação da microbiota pode ser útil para o tratamento da doença. Objetivo: compilar os principais resultados de ensaios clínicos randomizados sobre a utilização de bifidobactérias e lactobacilos no tratamento da colite ulcerativa. Metodologia: trata-se de uma revisão sistemática que utilizou as bases de dados Pubmed e Google Acadêmico para pesquisar artigos originais de ensaios clínicos randomizados em humanos, publicados entre 2003 e 2017, em inglês. Resultados: foram incluídos: dois ensaios multicêntricos, randomizados, duplo-cego; dois ensaios randomizados, duplo-cego; dois ensaios randomizados; e um ensaio randomizado aberto. O tratamento com L. delbruekii e L. fermentum melhorou a inflamação. O leite fermentado com B. breve, B. bifidum e L. acidophilus e a suplementação L. rhamnosus estirpe $G G$ melhoraram os sintomas clínicos, chegando a superar o tratamento padrão. A cepa $B$. longum induziu a remissão. E o leite fermentado com B. breve e $L$. acidophilus manteve a remissão da doença. Conclusão: embora, a maior parte dos resultados tenha sido positiva, algumas limitações reduzem a aplicabilidade desses achados em outras populações, como o desenho experimental, o tamanho da amostra e a diversidade de cepas probióticas. Por isso, novos ensaios clínicos randomizados, controlados e com populações maiores devem ser realizados.
\end{abstract}

Palavras-chave: Bifidobacterium. Lactobacillus. Colite ulcerativa.

\begin{abstract}
Introduction: ulcerative colitis is a chronic disease, which causes inflammation of the mucous membranes of the colon, cecum and rectum. Some evidence suggests that colitis development and recurrence are related to genetic susceptibility, intestinal microbiota imbalance and abnormal response of the immune system to enteric antigens. In view of this, modulation of the microbiota may be useful for the treatment of the disease. Objective: to compile the main results of randomized clinical trials on the use of bifidobacteria and lactobacilli in the treatment of ulcerative colitis. Methodology: this is a systematic review using the Pubmed and Google Scholar databases for original articles from randomized clinical trials in humans published between 2003 and 2017 in English. Results: two double-blind, multicenter, randomized trials were included; two randomized, double-blind trials; two randomized trials; and an open randomized trial. Treatment with L. delbruekii and L. fermentum improved inflammation. The milkfermented with B. breve, B. bifidum and L. acidophilus and L. rhamnosus strain GG supplementation improved the clinical symptoms, even surpassing the standard treatment. B. longum strain induced remission. And the milk fermented with B. breve and L. acidophilus maintained remission of the disease. Conclusion: although most of the results were positive, some limitations reduce the applicability of these findings in other populations, such as experimental design, sample size and diversity of probiotic strains. Therefore, new, randomized, controlled trials with larger populations should be performed.
\end{abstract}

Keywords: Bifidobacterium. Lactobacillus. Ulcerative colitis.

\section{INTRODUÇÃO}

As doenças inflamatórias intestinais (DIIs) são distúrbios idiopáticos e reincidentes, caracterizados pela presença de inflamação crônica em todo o trato gastrointestinal (TGI) ou parte dele (SARTOR, 2006). Os principais fenótipos das DIls são a doença de Crohn (DC)

Correspondente/ Corresponding: *Marcos da Costa Silva - Departamento de Ciências da Vida, Universidade do Estado da Bahia - End: Rua Silveira Martins - Cabula. CEP: 41150000 . Salvador/BA - Tel: (71) 3117-2200 -E-mail:marcosilva@uneb.br e a colite ulcerativa (RCU) (BOLDEANU et al., 2014). A DC pode acometer todo o TGI, mas geralmente afeta o íleo terminal e o cólon; a inflamação é transmural e segmentar (MATRICON; BARNICH; ARDID, 2010). A RCU é restrita ao cólon, ceco e reto, e as lesões patológicas são tipicamente limitadas à mucosa em um padrão contínuo (RONNBLOM; SAMUELSSON; EKBOM, 2010). Ambas têm manifestações clínicas semelhantes, que incluem diarreia severa, dor abdominal, fadiga e perda de peso (BOLDEANU et al., 2014). A patogênese das DIls não está completamente elucidada, mas se acredita que envolve uma interação 
complexa entre genética, sistema imunológico (SI), ambiente e o ecossistema entérico (CHEN; REN; SHI, 2014).

A microbiota intestinal humana contém entre $10^{11}$ e $10^{12}$ micro-organismos, o que inclui bactérias, fungos, protozoários e vírus. As bactérias são os micro-organismos dominantes, em adultos; a maioria delas pertence aos Filos Firmicutes (gram-positivas) e Bacteroidetes (gram-negativas), e a minoria aos Filos Actinobacteria e Proteobacteria (MILLION et al., 2013; PINTO, 2016; SANTOS; CONCEIÇÃO; SILVA, 2018; THOMAS, 2011). Em condições fisiológicas, esses organismos são inofensivos ao homem e têm papel importante na nutrição, na imunorregulação e na proteção do epitélio intestinal (SILVA et al., 2016). Quando há um desequilíbrio entre bactérias comensais e patogênicas (disbiose), a composição da microbiota intestinal é alterada em função do supercrescimento de bactérias nocivas (HEMAISWARYA et al., 2013). A disbiose favorece a invasão da mucosa e leva a uma maior ativação do SI, a ponto de causar uma resposta inflamatória exacerbada, que poderá contribuir para o surgimento de DIIs, como enterocolite necrosante, síndrome do intestino irritável, RCU, DC e doenças autoimunes (SHEN et al., 2018; XU et al., 2015).

A etiopatogenia da RCU é complexa, mas é possível que a microbiota intestinal seja um dos fatores-chave para o seu início e desenvolvimento (SHEN et al., 2018). Pacientes com RCU apresentam diversas alterações na microbiota, que incluem declínio da população de lactobacilos e bifidobactérias, e um aumento de Escherichia coli, coliformes e Bacteroidetes (HANSEN; GULATI; SARTOR, 2010; SAEZ-LARA et al., 2015). O crescimento dessas últimas bactérias foi relacionado às lesões nos tecidos intestinais e à progressão da doença (CONTE et al., 2006; KUWAHARA et al., 2004; URONIS et al., 2009). Por isso, pode ser de fundamental importância para o tratamento da RCU a modulação da microbiota intestinal (ZHANG; WANG; MIAO, 2017).

Para esse propósito, os probióticos apresentam potencial terapêutico promissor, pois são capazes de meIhorar as condições associadas à RCU (SHEN et al., 2018). Probióticos são micro-organismos vivos que, em concentrações adequadas, promovem benefícios à saúde, como equilíbrio da população de micro-organismos entéricos e melhora da imunidade local e sistêmica (KINROSS; DARZI; NICHOLSON, 2011; SANTOS; CONCEIÇÃO; SILVA, 2018). Os gêneros de bactérias mais utilizados como probióticos são Lactobacillus e Bifidobacterium (SAEZ-LARA et al., 2015). Embora diversos artigos de revisão abordem os efeitos das bifidobactérias e dos lactobacilos na RCU, poucos estudos consideram apenas a resposta dessas espécies, pois, geralmente, elas estão associadas com outros micro-organismos ou fibra alimentar. Por isso, um maior entendimento da ação e da relação desses dois grandes grupos de micro-organismos na RCU torna-se importante. Para uma melhor compreensão dessa relação, esta revisão teve como objetivo compilar os principais resultados de ensaios clínicos randomizados sobre a utilização de bifidobactérias e lactobacilos no tratamento da RCU.

\section{METODOLOGIA}

Este estudo consiste em uma revisão sistemática, que utilizou as bases de dados Pubmed e Google Acadêmico, pois elas disponibilizaram um maior número de artigos relacionados ao tema. Também foram incluídos trabaIhos encontrados em outras bases de dados, como Lilacs e Scientific Eletronic Library Online (SciELO). Para essa pesquisa, foram utilizados, em inglês, os termos MeSH Database: "Bifidobacterium" e "Lactobacillus, combinados com "ulcerative colitis". Foram considerados artigos originais de ensaios clínicos randomizados em humanos, publicados entre 2003 e 2018, período correspondente à presença de artigos que atendiam aos critérios de inclusão e exclusão estabelecidos. Não foram incluídos artigos de revisão, resumos e estudos realizados com outras doenças, simbióticos, outras espécies de bactérias e ensaios que tenham usado a via retal para administração dos probióticos.

Inicialmente, foram selecionados 55 estudos, e se excluíram as duplicações. Os artigos remanescentes foram triados por título, resumo e texto completo. Apenas artigos com resultados específicos sobre as bifidobactérias e os lactobacilos no tratamento da RCU foram considerados. A Figura 1 mostra o fluxo de seleção dos artigos.

\section{Figura 1 - Fluxograma da elegibilidade dos artigos}

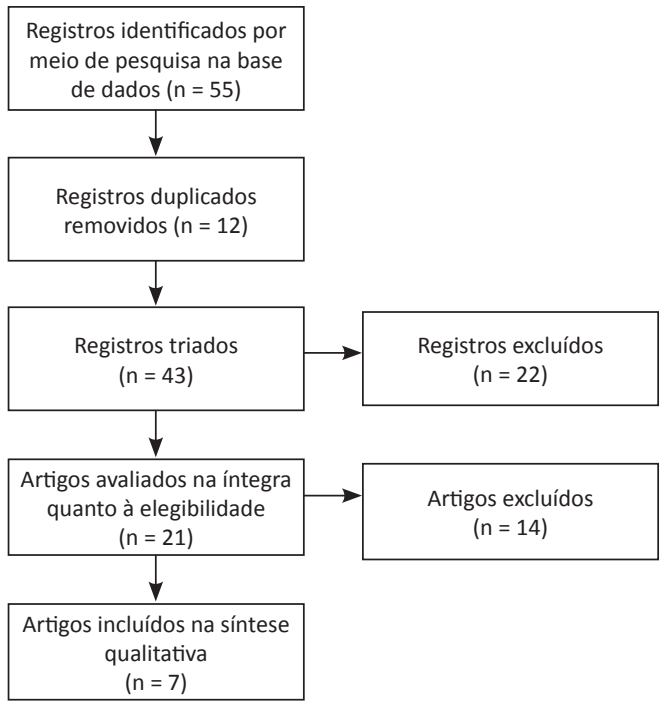

Fonte: Autoria própria.

\section{RESULTADOS}

Dentre os sete artigos selecionados, três avaliaram a ação dos probióticos no tratamento da RCU em fase ativa. Kato et al. (2004) compararam o efeito do leite fermentado com $B$. breve, B. bifidum e $L$. acidophilus ao da Mesalazina (5-ASA) ou Salazosulfapiridina (SASP). Primariamente, a eficácia dos tratamentos foi determinada pela redução de pelo menos três pontos no Índice de Atividade Clínica (CAI) em 12 semanas. O CAI reflete 
o padrão de evacuação, dor, cólicas abdominais, bem-estar geral e uso de antidiarreicos; escores altos indicam pior prognóstico. Em geral, os tratamentos foram bem tolerados. O grupo tratado com leite fermentado apresentou um CAl significativamente menor, pois cerca de $70 \%$ dos pacientes responderam ao tratamento e $40 \%$ apresentaram remissão clínica. No grupo controle, 33\% dos pacientes responderam ao 5-ASA e $33 \%$ alcançaram à remissão. Embora, a resposta ao probiótico tenha sido positiva, a extrapolação desses resultados é limitada, pois o estudo foi unicêntrico, e o tamanho da amostra e o tempo de seguimento foram pequenos.

O estudo multicêntrico, randomizado, duplo-cego conduzido por Tamaki et al. (2015) avaliou a ação do $B$. longum em induzir a remissão da RCU em pacientes japoneses através do Índice de Atividade de doença de Colite Ulcerosa (UCDAI), desfecho primário. Em oito semanas, houve diminuição significativa do UCDAI no grupo que recebeu a cepa probiótica, em comparação ao que usou o placebo. Os itens que compõem a UCDAl também foram investigados, e observou-se que pacientes tratados $\operatorname{com} B$. longum tiveram redução do sangramento retal e melhora da condição da mucosa, sendo que $29,2 \%$ apresentaram cicatrização das lesões, embora essa diferença não fosse estatisticamente significativa. Esses resultados têm baixa validade externa, pois a população estudada e o tempo de tratamento foram menores.

Hegazy e El-Bedewy (2010) analisaram o efeito dos $L$. delbruekii e $L$. fermentum na ativação do fator de transcrição NF-KB e na secreção de mediadores inflamatórios. Os pacientes foram estratificados em dois grupos: o primeiro recebeu sulfassalazina e o placebo, e o segundo sulfassalazina e os lactobacilos. Após oito semanas, o grupo que recebeu o medicamento padrão e $L$. delbruekii e $L$. fermentum apresentou redução significativa na expressão de NF-KB, interleucina 6 (IL-6), fator de necrose tumoral (TNF- $\alpha$ ) e mieloperoxidase (MPO), um índice de infiltração de leucócitos. Com esses resultados, os autores concluíram que os probióticos podem ser úteis para manter a remissão e evitar recidivas da RCU. Novamente, a aplicabilidade desses achados é restrita, por causa do pequeno número de pacientes, do tempo de seguimento baixo e por ter sido realizado em um único centro.

Alguns ensaios clínicos também verificaram a resposta à suplementação de bifidobactérias e lactobacilos no período de remissão da RCU. Nesta revisão, quatro artigos abordam esse tipo de intervenção. Um ensaio randomizado, feito por Ishikawa et al. (2003) testou o leite fermentado com B. breve e L. acidophillus como adjuvante no tratamento da RCU durante um ano. Os resultados mostraram que os pacientes suplementados com o probiótico tiveram uma redução nas exacerbações em comparação com o grupo de controle. Além do mais, a análise das fezes e, subsequentemente, da microbiota demonstrou que esses sujeitos também tiveram uma redução estatisticamente significativa da população de $B$. vulgatus. Embora as cepas probióticas tenham apre- sentado resposta favorável à manutenção da remissão, limitações referentes aos poucos participantes e ao desenho do estudo reduzem o poder externo dos resultados.

Zocco et al. (2006) compararam a eficácia do L. rhamnosus estirpe GG sozinho e associado com 5-ASA e 5-ASA isoladamente. $O$ desfecho primário consistiu em avaliar a manutenção da remissão, e isso foi determinado por um CAI menor que 4 em 12 meses. Após seis e 12 meses de tratamento, a remissão foi mantida em 91 e $85 \%$ dos pacientes que receberam apenas L.GG; 87 e $80 \%$ dos que receberam somente 5-ASA; e 94 e $84 \%$ dos que receberam a terapia combinada. A recidiva da doença ocorreu em 10 dos 65 sujeitos tratados com L. GG, 12 dos 60 sujeitos tratados com 5-ASA, e 10 dos 62 sujeitos tratados com a L.GG e 5-ASA. De modo geral, o efeito do L.GG, sozinho ou combinado, foi efetivo. Mas, entre o probiótico isolado e a terapia combinada, não houve diferença significativa, segundo os pesquisadores, e isso se deve a uma provável interação medicamentosa. Mais uma vez, o tamanho da população e o desenho do estudo, ensaio randomizado aberto, comprometeram a aplicabilidade dos resultados.

A suplementação com $B$. animalis subsp. lactis estirpe BB-12 e L. acidophilus estirpe LA-5 foi testada por Wildt et al. (2011), com o objetivo de avaliar a eficácia das cepas na manutenção da remissão da RCU esquerda, durante 52 semanas. Os pacientes incluídos no estudo tiveram de interromper o uso do 5-ASA. Na estratificação da amostra, 20 pacientes foram tratados com $B$. animalis subsp. lactis estirpe BB-12 e L. acidophilus estirpe LA-5; 12 pacientes receberam o placebo. Os resultados revelaram que $25 \%$ dos sujeitos tratados com o probiótico e $8 \%$ dos sujeitos do grupo placebo não apresentaram exarcebações no período de 52 semanas, mas não houve diferença significativa entre esses grupos. É provável que o pequeno número de pacientes envolvidos tenha interferido na detecção de uma possível diferença estatística. Além disso, a execução em um único centro diminui a validade externa.

Matsuoka et al. (2018) também avaliaram o efeito do leite fermentado com B. breve e $L$. acidophillus para prevenção de exarcebações da RCU, em um ensaio multicêntrico, randomizado, duplo-cego, controlado por placebo. $O$ desfecho primário foi estabelecido pela ausência de recidivas. A recidiva foi determinada pelo escore de sangramento retal $\geq 2$ na escala de Atividade da Doença de Sutherland (DAI) por três dias consecutivos e (ou) início da terapia de indução da remissão. O leite fermentado e a bebida placebo foram entregues nas residências dos pacientes. Os resultados demonstraram que nem o grupo tratado nem o grupo placebo apresentaram diferença estatística na remissão da doença em 48 semanas. No desfecho secundário, também não houve diferença no tempo de recidiva e nas médias dos escores de DAl, sintomas abdominais, frequência de evacuações, sangramento retal ou classificação da atividade da doença pelo médico. Os autores sugeriram que o método utilizado para entrega das bebidas ou a concentração das cepas probióticas tenham influenciado no resultado. 
O Quadro 1 apresenta mais detalhes sobre os estudos

e seus principais resultados.

Quadro 1 - Síntese dos ensaios clínicos que investigaram a eficácia das bifidobactérias e lactobacilos no tratamento da RCU.

\begin{tabular}{|c|c|c|c|c|c|c|c|}
\hline Referência & Tipo de estudo & $\begin{array}{l}\text { Número de } \\
\text { pacientes }\end{array}$ & $\begin{array}{l}\text { Idade dos } \\
\text { pacientes }\end{array}$ & $\begin{array}{l}\text { Características } \\
\text { da doença }\end{array}$ & Intervenção & Dose / Tempo & Resultado principal \\
\hline $\begin{array}{l}\text { Ishikawa et } \\
\text { al., } 2003 .\end{array}$ & Randomizado. & 21 & $39-60$ anos & $\begin{array}{l}\text { RCU em remis- } \\
\text { são clínica. }\end{array}$ & $\begin{array}{l}\text { Leite fermentado } \\
\text { com B. breve } 10^{10} \\
\text { UFC e L. acido- } \\
\text { phillus } 10^{9} \text { UFC. }\end{array}$ & $\begin{array}{l}100 \mathrm{~mL}-1 \mathrm{x} / \mathrm{dia} \text {, } \\
\text { por } 12 \text { meses. }\end{array}$ & $\begin{array}{l}\text { A remissão da doen- } \\
\text { ça foi mantida. }\end{array}$ \\
\hline
\end{tabular}

Kato et al., Randomizado, du- 20 $2004 . \quad$ plo-cego, controlado por placebo.

Zocco et al., Randomizado aber- 187 $2006 . \quad$ to

Hegazy e Randomizado. $\quad 30$ El-Bedewy, 2010.

Wildt et al. 2011.

Tamaki et al., 2015. domizado, duplo-cego, controlado por placebo.

Matsuoka et Multicêntrico, ran- 195 al., 2018.
Randomizado, du- 32 plo-cego, controlado por placebo.
Média de RCU ativa, leve a Leite fermenta- $100 \mathrm{~mL}-1 x /$ dia, A suplementação fo 31,9 anos moderada. do com $B$. breve por 12 semanas. mais efetiva que o estirpe Yakult, B. bifidum e $L$. acidophilus $10^{10}$ UFC.

Média de 33 RCU em remis- L. rhamnosus es- 2x/dia, por 12 Mesma eficácia que anos são clínica. tirpe $G G 6 \times 10^{9}$ meses. a mesalazina para UFC. tratamento convencional. manter a remissão mas significativa mente melhor no retardo de recidivas.

Média de 47 RCU ativa, levea L. delbruekii e L. 1x/dia, por 8 se- Melhora da inflamaanos moderada. fermentum $10^{10}$ manas. ção. Pode contribuir UFC. para prevenção de recidivas e manutenção da remissão.

23-68 anos RCU em remis- B. animalis subsp. Duas cápsulas Nenhum efeito comsão clínica. lactis B B-12-3x/dia, por 52 parado como grupo $1,25 \times 10^{10}$ UFC $L$. semanas. placebo. acidophilus LA-5 $1,25 \times 10^{10}$ UFC.

Maiores de 18 RCU ativa, levea B. longum 536 3x/dia, por oito Remissão da doença

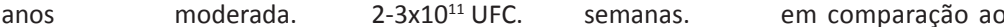
grupo placebo.

20-70 anos RCU em remis- Leite fermentado $100 \mathrm{~mL}-1 \mathrm{x} /$ dia, Nenhum efeito comsão clínica. $\quad \operatorname{com} B$. breve es- por 48 semanas. parado com o grupo tirpe Yakult $10^{10}$ UFC e L. acidoplacebo.

-cego, controlado por placebo.

\section{DISCUSSÃO}

A RCU é uma doença inflamatória crônica, que envolve aumento da permeabilidade intestinal, edema epitelial e vasta infiltração de leucócitos (HEGAZY; EL-BEDEWY, 2010). É caracterizada por períodos de exacerbação e períodos de remissão (SAEZ-LARA et al., 2015). A fisiopatologia inclui desregulação da resposta do $\mathrm{SI}$ a antígenos do lúmen intestinal, como as bactérias (ISHIKAWA et al., 2011; MATSUOKA et al., 2018; TOJO et al., 2014). O papel da microbiota intestinal na RCU foi demonstrado em modelos experimentais; animais germ free não desenvolvem ou não apresentam progressão significativa da doença (HILL; DRASAR, 1975; MIZOGUCHI; NGUYEN; LOW, 2013). Na RCU, a inflamação é influenciada pela permeabilida- de intestinal aumentada, susceptibilidade genética do hospedeiro e pelo contato direto com as bactérias ou seus metabólitos (HEGAZY; EL-BEDEWY, 2010; SOMMER; BACKHED, 2013).

Tradicionalmente, o tratamento da RCU consiste em induzir a remissão. Isso normalmente é feito por meio da prescrição de anti-inflamatórios, corticosteroides (tópicos e sistêmicos) e anti-TNF- $\alpha$ (MIELE et al., 2009). Esses fármacos diminuem a lesão e a produção de alguns mediadores inflamatórios. Entretanto muitos pacientes têm efeitos colaterais e, em longo prazo, não toleram a terapia (HEVIA et al., 2015; KATZ, 2002). Nos últimos anos, tem sido destacado que os probióticos são capazes de restaurar a microbiota por aumentar a biodiversidade 
e melhorar os sintomas da RCU, devido à supressão da cascata inflamatória (TOJO et al., 2014). Para esse fim, as cepas mais usadas são as bifidobactérias e os lactobacilos (HEVIA et al., 2015). Essas bactérias podem inibir competitivamente a adesão de patógenos e regular o SI a ponto de estimular a liberação de citocinas anti-inflamatórias, como interleucina 10 (IL-10) (ESAIASSEN et al., 2017; SAEZ-LARA et al., 2015).

O efeito das bifidobactérias e dos lactobacilos no tratamento da RCU foi investigado por alguns ensaios clínicos. Nesta revisão, apenas um estudo avaliou o efeito dos probióticos especificamente sobre a resposta imunológica. O tratamento com L. delbruekii e L. fermentum, combinados com a sulfassalazina, resultou na diminuição significativa da expressão de NF-kB, IL-6, TNF- $\alpha$ e MPO, o que pôde contribuir para indução e manutenção da remissão (HEGAZY; EL-BEDEWY, 2010). O NF-kB é um fator de transcrição que regula a expressão de diversos genes, incluindo genes anti-apoptóticos, que reprimem a apoptose de neutrófilos e macrófagos ativados, o que contribui para extensão e agravamento da lesão tecidual. Além do mais, pode controlar a liberação de citocinas pró-inflamatórias, incluindo IL-6 e TNF- $\alpha$ (BENGOECHEA-ALONSO et al., 2003). O TNF- $\alpha$ tem um papel essencial na patogênese da RCU (MOZES et al., 2012). Seus efeitos envolvem estímulo às respostas citotóxicas, apoptóticas e de fase aguda. Também induz a liberação de outras citocinas, como IL-1ß, IL-6 e IL-33 (SANCHEZ-MUNOZ; DOMINGUEZ-LOPEZ; YAMAMOTO-FURUSHO, 2008). A sinalização da IL-6, via transdutor de sinais e ativador de transcrição 3 (STAT3), é importante para o desenvolvimento da RCU e neoplasias colorretais associadas (LI et al., 2009).

Os demais estudos investigaram a eficácia das cepas probióticas para induzir ou manter a remissão por meio de parâmetros clínicos. Na RCU exacerbada, a maior parte dos resultados indicou que os probióticos podem induzir a remissão (KATO et al., 2004; TAMAKI et al., 2015). Kato et al. (2004) mostraram que o leite fermentado com $B$. breve, $B$. bifidum e $L$. acidophilus foi mais efetivo do que a terapia padrão. Na RCU em remissão, os probióticos foram eficazes para manter o estado (ISHIKAWA et al., 2003; ZOCCO et al., 2006). Além disso, o L. GG foi superior a 5-ASA para retardar as recidivas (ZOCCO et al., 2006). Apenas dois ensaios não encontram diferença significativa entre os grupos. É provável que os probióticos exerçam seus efeitos por meio de três mecanismos: produção de lactato e ácidos graxos de cadeia curta (AGCC), que atuam como agentes anti-inflamatórios, modulam células imunes através de receptores Gpr43 e Gpr41, e impedem o crescimento de micro-organismos potencialmente patogênicos (HEVIA et al., 2015; ZOCCO et al., 2006); aumento do fluxo de água para a luz intestinal, o que altera a aderência das bactérias ao epitélio; e redução da produção de metabólitos tóxicos (SAADATZADEH et al., 2011; SAEZ-LARA et al., 2015).

Nesses estudos, não foram relatados efeitos adversos e contraindicações, mas não é possível presumir que essas cepas sejam igualmente adequadas a todos os portadores de RCU. Isso porque a maioria dos ensaios clínicos incluídos apresenta um curto período de seguimento, além de variações no tamanho amostral e nas metodologias. Entretanto os resultados apresentados demonstram que as bifidobactérias e os lactobacilos têm um potencial terapêutico promissor para RCU.

\section{CONCLUSÃO}

Esta revisão compilou alguns estudos de intervenção em humanos, demonstrando que as bifidobactérias e os lactobacilos, de modo geral, são eficazes para melhorar sintomas, induzir e manter o período de remissão da RCU. Contudo são resultados preliminares, cuja aplicabilidade em outras populações é limitada, pois foram obtidos em ensaios clínicos pequenos e heterogêneos quanto à definição de parâmetros clínicos para caracterização das fases da doença e composição dos probióticos utilizados. Por isso, outros estudos multicêntricos, randomizados e controlados com mais participantes devem ser realizados a fim de evidenciar as cepas probióticas mais eficazes e seus mecanismos de ação, consolidando o uso desses micro-organismos no tratamento da RCU.

\section{REFERÊNCIAS}

BENGOECHEA-ALONSO, M. T. et al. Regulation of NF-KB activation by protein phosphatase $2 \mathrm{~B}$ and $\mathrm{NO}$, via protein kinase $\mathrm{A}$ activity, in human monocytes. Nitric oxide, Orlando, v. 8, n. 1, p. 65-74, 2003.

BOLDEANU, M. V. et al. Investigation of inflammatory activity in ulcerative colitis. Rom. j. morphol. embryol., Bucuresti, p.1345-1351, 2014.

CHEN, W-X.; REN, L-H; SHI, R-H. Enteric microbiota leads to new therapeutic strategies for ulcerative colitis. World j. gastroenterology, Beijing, p. 15657-15663, 2014.

CONTE, M. P et al. Gut-associated bacterial microbiota in paediatric patients with inflammatory bowel disease. Gut, London, v. 55, n.12, p.1760-1767, 2006.

ESAIASSEN, E. et al. Bifidobacterium bacteremia: clinical characteristics and a genomic approach to assess pathogenicity. J. clin. microbiol., Washington, p.2234-2248, 2017.

HANSEN, J.; GULATI, A.; SARTOR, R. B. The role of mucosal immunity and host genetics in defining intestinal commensal bacteria. Curr. opin. gastroenterology., Philadelphia, v. 26, n. 6, p. 564-571, 2010.

HEGAZY, S. K.; EL-BEDEWY, M. M. Effect of probiotics on pro-inflammatory cytokines and NF-KB activation in ulcerative colitis. World j. gastroenterol., Beijing, v. 16, n. 33, p.4145-4151, 2010.

HEMAISWARYA, S. et al. Mechanism of action of probiotics. Braz. arch. biol. technol., Curitiba, v. 56, n. 1, p. 113-119, 2013.

HEVIA, A et al. Molecular players involved in the interaction between beneficial bacteria and the immune system. Front Microbiol., Lausanne, v. 6, p.1-8, 2015.

HILL, M. J.; DRASAR, B S. The normal colonic bacterial flora. Gut, London, v. 16, n. 4 , p. $318-323,1975$.

ISHIKAWA, H. et al. Randomized controlled trial of the effect of bifidobacteria-fermented milk on ulcerative colitis. J. Am. College Nutr., New York, v. 22, n. 1, p.56-63, 2003. 
ISHIKAWA, $\mathrm{H}$ et al. Beneficial effects of probiotic bifidobacterium and galacto-oligosaccharide in patients with ulcerative colitis: a randomized controlled study. Digestion, Basel, v. 84, n. 2, p.128-133, 2011.

KATO, K. et al. Randomized placebo-controlled trial assessing the effect of bifidobacteria-fermented milk on active ulcerative colitis. Aliment. pharmacol. ther., Oxford, v. 20, n. 10, p.1133-1141, 2004.

KATZ, J. A. Advances in the medical therapy of inflammatory bowel disease. Curr. opin. gastroenterol., Philadelphia, v.18, p. 435-440, 2002.

KINROSS, J. M.; DARZI, A. W.; NICHOLSON, J. K. Gut microbiome-host interactions in health and disease. Genome med., London, v. 3, n. 3, p.1-12, 2011.

KUWAHARA, T. et al. Genomic analysis of Bacteroides fragilis reveals extensive DNA inversions regulating cell surface adaptation. Proc. Natl. Acad. Sci. U. S. A., Washington, v. 101, n. 41, p.14919-14924, 2004.

LI, Yi et al. Disease-related expression of the IL6/STAT3/SOCS3 signalling pathway in ulcerative colitis and ulcerative colitis-related carcinogenesis. Gut, London, v. 59, n. 2, p.227-235, 2009.

MATRICON, J.; BARNICH, N.; ARDID, D. Immunopathogenesis of inflammatory bowel disease. Self Nonself, Austin, v. 1, n. 4, p.299-309, 2010.

MATSUOKA, K. et al. Efficacy of bifidobacterium breve fermented milk in maintaining remission of ulcerative colitis. Dig. dis. sci., New York, v. 63, n. 7, p. 1910-1919, 2018.

MIELE, E. et al. Effect of a probiotic preparation (VSL\#3) on induction and maintenance of remission in children with ulcerative colitis. Am. j. gastroenterology, New York, v. 104, n. 2, p. 437-443, 2009.

MILLION, M. et al. Gut bacterial microbiota and obesity. Clin. microbiol. infection, Oxford, v. 19, n. 4, p.305-313, 2013.

MIZOGUCHI, E.; NGUYEN, D.; LOW, D. Animal models of ulcerative colitis and their application in drug research. Drug Des. Devel. Ther., New Zealand, p.1341-1357. 2013.

MOZES, Gyorgyi. Changes of the cytokine profile in inflammatory bowel diseases. World Journal of Gastroenterology, Beijing, v. 18, n. 41, p.5848-5861, 2012.

PINTO, C. T. Homeostase da microbiota intestinal: saúde ou doença no homem. 2016. 27 f. Dissertação (Mestrado) - Curso de Ciências Farmacêuticas, Universidade de Coimbra, Coimbra, 2016.

RONNBLOM, A.; SAMUELSSON, S. M.; EKBOM, A. Ulcerative colitis in the county of Uppsala 1945-2007: incidence and clinical characteristics. J. crohns. colitis, Amsterdam, p.532-536, 2010.

SAADATZADEH, A. et al. Biochemical and pathological evidences on the benefit of a new biodegradable nanoparticles of probiotic extract in murine colitis. Fundam. clin. pharmacol., Paris, v. 26, n. 5, p.589-598, 2011.

SAEZ-LARA, M. J. The Role of probiotic lactic acid bacteria and bifidobacteria in the prevention and treatment of inflammatory bowel disease and other related diseases: a systematic review of randomized human clinical trials. Biomed Res. Int., New York, v. 2015, p.1-15, 2015.

SANCHEZ-MUÑOZ, F.; DOMINGUEZ-LOPEZ, A.; YAMAMOTO-FURUSHO, $J$. K. Role of cytokines in inflammatory bowel disease. World j. gastroenterol., Beijing, v. 14, n. 27, p.4280-4288, 2008.

SANTOS, L. A. dos; CONCEIÇÃO, E. dos S. da; SILVA, M. da C. Influência da microbiota intestinal na síndrome metabólica. In: ARAÚJO, E. M. Q. (Org.) A síndrome metabólica e suas implicações clínicas. Salvador: Eduneb, 2018. cap. 4. p. 113-138.

SARTOR, R. B. Mechanisms of disease: pathogenesis of Crohn's disease and ulcerative colitis. Nat. Clin. Pract. Gastroenterol. Hepatol., London, v. 7, n. 3, p. 390-407, 2006.

SHEN, Z-H et al. Relationship between intestinal microbiota and ulcerative colitis: Mechanisms and clinical application of probiotics and fecal microbiota transplantation. World j. gastroenterol., Beijing, v. 24, n. 1, p.5-14. 2018.

SILVA, F. A. R et al. The immunological basis of inflammatory bowel disease. Gastroenterology Research and Practice, London, v.2016, p.1-11. 2016.

SOMMER, F.; BACKHED, F. The gut microbiota-masters of host development and physiology. Nat. rev. microbiol., London, v.11, n.4, p.227-238, 2013.

TAMAKI, H. et al. Efficacy of probiotic treatment with Bifidobacterium longum 536 for induction of remission in active ulcerative colitis: A randomized, double-blinded, placebo-controlled multicenter trial. Dig. Endoscopy., Tokyo, v. 28, n. 1, p.67-74, 2015.

THOMAS, F et al. Environmental and gut bacteriodetes: the food connection. Front. Microbiol., Lausanne, p.1-16, 2011.

TOJO, R. et al. Intestinal microbiota in health and disease: Role of bifidobacteria in gut homeostasis. World j. gastroenterol., Beijing, p. 15163-15176, 2014.

URONIS, J. M. et al. Modulation of the intestinal microbiota alters colitis-associated colorectal cancer susceptibility. PLoS ONE, London, v.4, n.6, p.1-9, 2009.

WILDT, S. et al. A randomised double-blind placebo-controlled trial with Lactobacillus acidophilus La- 5 and Bifidobacterium animalis subsp. lactis BB-12 for maintenance of remission in ulcerative colitis. J. crohns. colitis, Amsterdam, v. 5, n. 2, p.115-121, abr. 2011.

$\mathrm{XU}, \mathrm{M} . \mathrm{Q}$ et al. Fecal microbiota transplantation broadening its application beyond intestinal disorders. World j. gastroenterol., Beijing, v.21, n.1, p.102-111, 2015.

ZHANG, S-L; WANG, S-N; MIAO, C-Y. Influence of Microbiota on Intestinal Immune System in Ulcerative Colitis and Its Intervention. Front. Immunol., Lausanne, v. 8, p.1-11. 2017.

ZOcCO, M. A. et al. Efficacy of Lactobacillus GG in maintaining remission of ulcerative colitis. Alimentary. pharmacol. ther., Oxford, v. 23, n. 11, p.1567-1574, 2006.

Submetido em: 20/05/2019

Aceito: 05/09/2019 